\title{
Inversion of lift-off distance and thickness for non-magnetic metal using eddy current testing
}

\author{
Xiaobai Meng, Mingyang Lu, Wuliang Yin, Abdeldjalil Bennecer*, Katherine J Kirk*
}

\begin{abstract}
For the electromagnetic eddy current testing, various methods have been proposed for reducing the lift-off error on the measurement of samples. In this paper, instead of eliminating the measurement error caused by the lift-off effect, an algorithm has been proposed to directly measure the lift-off distance between the sensor and non-magnetic conductive plates. The algorithm is based on a sample-independent inductance (SII) feature. That is, under high working frequencies, the inductance is found sensitive to the lift-off distance and independent of the test piece under an optimal single high working frequency $(43.87 \mathrm{kHz})$. Furthermore, the predicted lift-off distance is used for the thickness prediction of the non-magnetic conductive samples using an iterative method. Considering the eddy current skin depth, the thickness prediction is operated under a single lower frequency $(0.20 \mathrm{kHz})$. As the inductance has different sensitivities to the liftoff and thickness, the prediction error of the sample thickness is different from that of the lift-off distance. From the experiments on three different nonmagnetic samples - aluminium, copper, and brass, the maximum prediction error of the lift-off distance and sample thickness is $1.1 \mathrm{~mm}$ and $5.42 \%$ respectively at the lift-off of $12.0 \mathrm{~mm}$.
\end{abstract}

Index Terms - Eddy current sensor, lift-off measurement, thickness measurement, non-destructive testing, sampleindependence.

\section{INTRODUCTION}

$\mathrm{V}$ ARIOUS techniques including the electromagnetic, ultrasonic, acoustic and laser sensing methods have been introduced in the field of the non-destructive testing (NDT) or non-destructive evaluation (NDE) [1-6]. Owing to its high adaptability, electromagnetic (EM) sensing has been massively used for the detection and measurement of surface fatigue information, quality inspection, and reconstruction of sample parameters (including the magnetic permeability, electrical conductivity, and thickness profiles) [7-12].

The magnetic flux leakage (MFL), eddy current testing (ECT) and permanent magnet perturbation (PMP) are currently the three dominant methods of EM NDT [13]. PMP methods have advantages of easily installed sensor but can only be applied in the measurement of ferromagnetic materials [13]. Besides, PMP methods are significantly sensitive to the lift-off distance to the test magnetic samples. MFL is playing an increasing role in the metallic quality check, detecting and characterising defects [14], particularly for the safety inspection

X. Meng, A. Bennecer, K. J. Kirk are with the Faculty of Art, Science and Technology, University of Northampton, Northampton, NN1 5PH, United Kingdom

M. Lu (Member, IEEE), W. Yin (Senior Member, IEEE) are with the School of Electrical and Electronic Engineering, University of Manchester, Sackville Street Building, Manchester, M13 9PL, United Kingdom of tubes and railway. Udpa and Kim have proposed several methods of improving the magnetic flux leakage methods in the remote region of the pipeline inspection [15-18].

ECT methods are generally applied for both magnetic and non-magnetic conductive materials. Diverse methods including the multi-frequency eddy current (MEC) sensing [20-29], pulsed eddy current (PEC) sensing [30-37], and numerical models $[38,39]$ have been used for the measurement of surface crack information (using rotating field) [40,41], thickness, magnetic permeability and electrical conductivity of the specimen. For the MEC technique, Pinotti and Puppin have proposed a lock-in approach to retrieve both the electrical conductivity and thickness of the specimen by referring to the voltage phase feature [22]. Different methods have been proposed to eliminate the lift-off effect on the thickness measurement of the nonmagnetic conductive plates $[12,19,23$, 29]. However, these methods are mainly focused on peak frequency feature from the inductance spectrum.

In this paper, an eddy current (EC) sensor with two planar and co-axial coils has been used to directly reconstruct the liftoff distance and measure the thickness of the non-magnetic conductive plate. The selected EC sensor is less mechanically complicated and more accurate than conventional triple-coil sensors without concerning the error of the magnetic balance. Although the used EC sensor was originally designed decades ago, the previous technique on the lift-off retrieval is based on the calibration method - Lissajous diagram [44] or iterative method on the conventional analytical model [37], whereas the proposed technique is using the thin-skin regime via simplified model (which only needs single frequency for the lift-off restoration, and applies for the online measurement). Based on this EC sensor, a lift-off algorithm has been proposed, which originates from a sample-independent inductance (SII) feature under high working frequencies. That is, under an optimal high frequency $-43.87 \mathrm{kHz}$ (for the sensor size used in this paper), the inductance is found sensitive to the lift-off distance and nearly immune to the test piece due to the (surface-restrained) eddy current skin effect. The error of the reconstructed lift-off reaches its smallest value $8.8 \mu \mathrm{m}$ at $2.0 \mathrm{~mm}$ and highest value $1.1 \mathrm{~mm}$ at $12.0 \mathrm{~mm}$. Furthermore, by using an iterative method, the predicted lift-off is used for the thickness prediction of different non-magnetic samples under a lower single frequency.

Corresponding authors are listed by alphabetical order:

A. Bennecer (Abdeldjalil.Bennecer@northampton.ac.uk)

K. J. Kirk (Katherine.Kirk@ northampton.ac.uk) 
The error of the predicted sample thickness achieves its lowest percentage $(0.11 \%)$ at the lift-off distance of $2.0 \mathrm{~mm}$, and highest one $(5.42 \%)$ at the lift-off distance of $12 \mathrm{~mm}$. Owing to the different sensitivity of the inductance regarding to the liftoff distance, the prediction error of the thickness differs from that of the lift-off distance.

\section{METHODOLOGY - PREDICTION ALGORITHMS}

\section{A. Original formula of circular coil above conductive plate}

Previously, different structures of eddy current sensors have been proposed for the lift-off compensation and thickness measurement [12,19,23,29], which mainly involves combining the measured signals from dual-sensing pairs (with three coils). In this paper, a simplified high-frequency regime model is extracted from a simple sensor setup (Fig. 1).

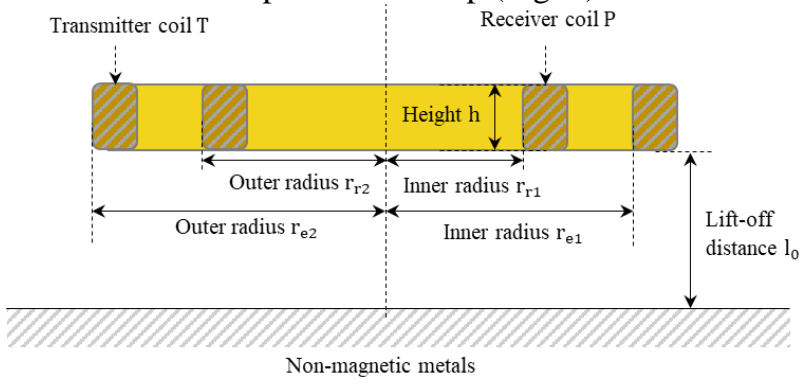

Fig. 1 Structure of two co-axial coils eddy current sensor

As shown in Fig. 1, the inductance change (minus the background signal when the sensor is in the free space) for two co-axial circular coils parallel but lifted with a small distance to the non-magnetic conductive plate can be derived from the Dodd-Deeds formulation [43],

$$
\mathrm{L}=\mathrm{K} \int_{0}^{\infty} \mathrm{Me}^{-2 \alpha \mathrm{l}_{0}} \mathrm{Td} \alpha
$$

Where in (1), $\mathrm{l}_{0}$ is the lift-off distance between the eddy current sensor and test piece. $\alpha$ is the integration variable related to the wavenumber of the incident transverse electric (TE) plane EM wave [43]. $\mathrm{M}$ and $\mathrm{T}$ are the magnitude part and phase part of the integrand for the mutual inductance, which are determined by the sensor geometry and test piece respectively. $\mathrm{M}$ is defined as

$$
M=\frac{P(\alpha)}{\alpha^{6}}\left(e^{-\alpha h}-1\right)^{2}
$$

In (2), $h$ is the height of the coils. $\mathrm{P}$ is the integration of the series of $\mathrm{J}_{1}$ over the exciting and receiver coils.

$$
P(\alpha)=\int_{\alpha r_{e 1}}^{\alpha r_{e} 2} \tau J_{1}(\tau) d \tau \int_{\alpha r_{r 1}}^{\alpha r_{r 2}} \tau J_{1}(\tau) d \tau
$$

In (3), $r_{\mathrm{e} 2}$ and $\mathrm{r}_{\mathrm{e} 1}$ are the outer and inner radii of the exciting coil, while $r_{r 2}$ and $r_{r 1}$ are that of the receiver coil. $J_{1}$ is the firstorder Bessel function of the first kind. $\tau$ is the integration variable. In (1), $\mathrm{K}$ is a constant factor related to the geometry of the sensor and defined as

$$
K=\frac{\pi\left(r_{r 1}+r_{r 2}\right) \mu_{0} N_{e} N_{r}}{2 h^{2}\left(r_{e 2}-r_{e 1}\right)\left(r_{r 2}-r_{r 1}\right)}
$$

where $\mu_{0}$ is the vacuum magnetic permeability. $\mathrm{N}_{\mathrm{e}}$ and $\mathrm{N}_{\mathrm{r}}$ are the number of turns of the exciting and receiver coils. The phase term $\mathrm{T}$ in (1) is defined as

$$
\mathrm{T}=\operatorname{Re}\left(\frac{\left(\alpha_{1}+\alpha\right)\left(\alpha_{1}-\alpha\right)-\left(\alpha_{1}+\alpha\right)\left(\alpha_{1}-\alpha\right) \mathrm{e}^{2 \alpha_{1} c}}{-\left(\alpha_{1}-\alpha\right)\left(\alpha_{1}-\alpha\right)+\left(\alpha_{1}+\alpha\right)\left(\alpha_{1}+\alpha\right) \mathrm{e}^{2 \alpha_{1} c}}\right)
$$

In (5), $c$ is the thickness of the non-magnetic test piece; $\alpha_{1}$ is a complex number related to the wavenumber of the transmitted EM TE plane wave, which is defined as

$$
\alpha_{1}=\sqrt{\alpha^{2}+\mathrm{j} 2 \pi \sigma \mu_{0} f}
$$

where $\sigma$ is the electrical conductivity of the test piece; $f$ is the working frequency of the current flowing in the exciting coil.

\section{B. Prediction of lift-off distance from quiescent inductance using simplified algorithm - high frequency-regime}

As shown in Fig. 2, it has been found that the magnitude part of the integrand $M$ can be approximated using $\sin ^{2}\left(\frac{\alpha \pi}{2 \alpha_{0}}\right)$, where $\alpha_{0}$ is related to the height and the radii of the coils.

$$
\mathrm{M}=S \sin ^{2}\left(\frac{\alpha \pi}{2 \alpha_{0}}\right)
$$

In (7), $S$ is the ratio factor between $\frac{\mathrm{P}(\alpha)}{\alpha^{6}}\left(\mathrm{e}^{-\alpha \mathrm{h}}-1\right)^{2}$ and $\sin ^{2}\left(\frac{\alpha \pi}{2 \alpha_{0}}\right)$.

$$
S=\frac{\mathrm{P}\left(\alpha_{0}\right)}{\alpha_{0}^{6}}\left(\mathrm{e}^{-\alpha_{0} \mathrm{~h}}-1\right)^{2}
$$

It can be observed from (8) that the ratio factor $S$ is a coildependent constant. Substituting (7) into (1), the inductance in (1) becomes,

$$
\mathrm{L}=\mathrm{KS} \int_{0}^{2 \alpha_{0}} \sin ^{2}\left(\frac{\alpha \pi}{2 \alpha_{0}}\right) \mathrm{e}^{-2 \alpha \mathrm{l}_{0}} \mathrm{Td} \alpha
$$

In (9), since the effective range of $\alpha$ for $M$ is from 0 to $2 \alpha_{0}$, the upper limit of the integration becomes $2 \alpha_{0}$.

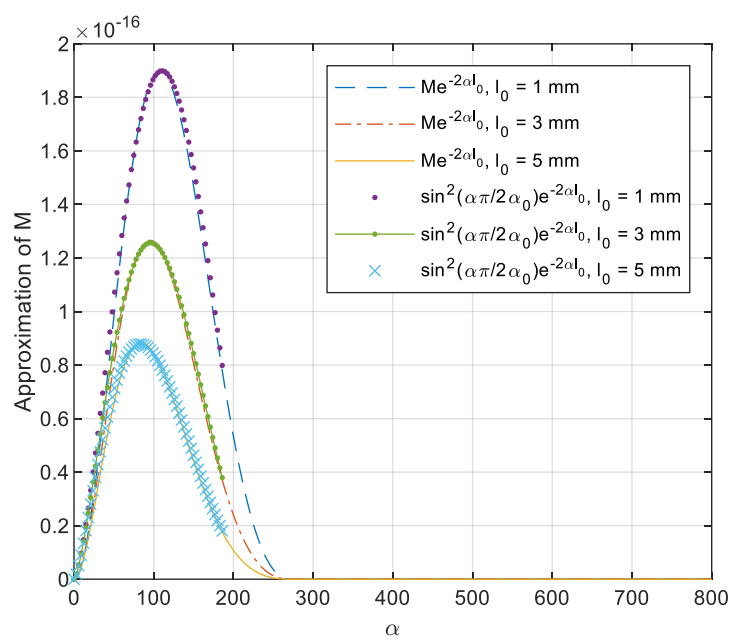

Fig. 2 Estimation for the magnitude part of the integrand - M under different lift-off distances

Since the eddy current is confined to the surface of the test piece under the high working frequency, the thickness of the sample barely affects the signal. That is, $\operatorname{Re}\left(\mathrm{e}^{2 \alpha_{1} c}\right) \geq 1$. Therefore, the phase part of the integrand - $\mathrm{T}$ in (5) can be estimated as

$$
\mathrm{T}=-\operatorname{Re}\left(\frac{\alpha_{1}-\alpha}{\alpha_{1}+\alpha}\right)
$$

Besides, it can be seen from Fig. 2 that, the maximum effective $\alpha$ for $\mathrm{M}$ is $2 \alpha_{0}$ (around 250 for the sensor in Table 1). Consequently, $\alpha^{2} \ll 2 \pi \sigma \mu_{0} f$ holds under high working frequencies. Then, $\mathrm{T}$ can be approximated as

$$
\mathrm{T}=-\operatorname{Re}\left(\frac{\sqrt{\pi \sigma \mu_{0} f}+\mathrm{j} \sqrt{\pi \sigma \mu_{0} f}-\alpha}{\sqrt{\pi \sigma \mu_{0} f}+\mathrm{j} \sqrt{\pi \sigma \mu_{0} f}+\alpha}\right)
$$


Since $\sqrt{\pi \sigma \mu_{0} f} \gg \alpha$, equation (11) becomes

Substituting (12) into (9), the inductance under the high working frequency becomes

$$
\mathrm{L}=-\mathrm{K} S \int_{0}^{2 \alpha_{0}} \mathrm{e}^{-2 \alpha \mathrm{l}_{0}} \sin ^{2}\left(\frac{\alpha \pi}{2 \alpha_{0}}\right) \mathrm{d} \alpha
$$

After the integration, (13) becomes

$$
\mathrm{L}=-\mathrm{KS} \frac{\pi^{2}\left(1-\mathrm{e}^{-4 \alpha_{0} \mathrm{l}_{0}}\right)}{4 \mathrm{l}_{0}\left(4 \alpha_{0}^{2} 1_{0}^{2}+\pi^{2}\right)}
$$

The lift-off of the eddy current sensor - $l_{0}$ can be derived by finding the numerical solution of the non-linear equation (14). It can be observed that under high working frequencies, the inductance value is (almost) immune to the test piece. Moreover, the solution of the lift-off is merely determined by the parameters of the eddy current sensor (including $\mathrm{h}$ and spatial frequency $\alpha_{0}$ - determined by the height $h$, and radii $r_{r 1}$, $\mathrm{r}_{\mathrm{r} 2}, \mathrm{r}_{\mathrm{e} 1}, \mathrm{r}_{\mathrm{e} 2}$ of coils).

\section{Prediction of sample thickness}

The derived lift-off, $\mathrm{l}_{0}$, from equation (14) can be further used for the prediction of the thickness of non-magnetic conductive plates. The thickness can be reconstructed with the modified Newton-Raphson method in an iterative manner. Parameters including the predicted lift-off, $\mathrm{l}_{0}$, the electrical conductivity, $\sigma$, and a lower single frequency are served as input for the prediction in each iterative loop. The single working frequency should be lower enough that the actual thickness of the test piece is much smaller than the skin depth computed by the following equation.

$$
\delta=\frac{1}{\sqrt{\pi \sigma \mu_{0} f}}
$$

The predicted thickness under the current iterative loop is

$$
c=c_{r}+\Delta c
$$

In (16), $c_{r}$ is the reference value of the thickness, i.e. the predicted thickness from the last iterative loop. For the first iterative loop, $c_{r}$ can be roughly predicted from the intuitive observations. $\Delta \mathrm{c}$ is the predicted change of the thickness and can be calculated as

$$
\Delta c=\mathrm{J}\left(\mathrm{L}_{\mathrm{m}}-\mathrm{L}\left(c_{r}\right)\right)
$$

In (17), $\mathrm{L}_{\mathrm{m}}$ is the measured inductance. $\mathrm{L}\left(c_{r}\right)$ is the computed inductance with the input of $c_{r}$ using equation (1). J is the reciprocal of the inductance sensitivity with respect to the thickness of the test piece $c_{r}$.

$$
\mathrm{J}=\frac{c_{r}-c_{0}}{\mathrm{~L}\left(c_{r}\right)-\mathrm{L}\left(c_{0}\right)}
$$

In (18), $c_{0}$ is a value very close to $c_{r}$ (it can be defined as $\left.c_{0}=0.95 c_{r}\right) . \mathrm{L}\left(\mathrm{c}_{0}\right)$ is the computed inductance with the input of $c_{0}$ using equation (1). The iterative loop can be stopped as $\frac{\Delta c}{c_{r}}$ approaches a small residual.

TABLE I

PARAMETERS OF EDDY CURRENT SENSOR

\begin{tabular}{ccc}
\hline \hline & Exciting coil & Receiver coil \\
\hline Inner radii $-\mathrm{r}_{\mathrm{e} 1}$ or $\mathrm{r}_{\mathrm{r} 1} / \mathrm{mm}$ & 19.7 & 14.4 \\
Outer radii $-\mathrm{r}_{\mathrm{e} 2}$ or $\mathrm{r}_{\mathrm{r} 2} / \mathrm{mm}$ & 13.2 & 13.9 \\
Height $-\mathrm{h} / \mathrm{mm}$ & \multicolumn{2}{c}{5.0} \\
Turns & \multicolumn{2}{c}{30} \\
Lift-off distance $-\mathrm{l}_{0} / \mathrm{mm}$ & \multicolumn{2}{c}{$1.0-12.0$} \\
\hline \hline
\end{tabular}

\section{EFFECT ANALYSIS AND MEASUREMENT SETUP}

\section{A. Analysis of measurement uncertainty - effect of thickness}

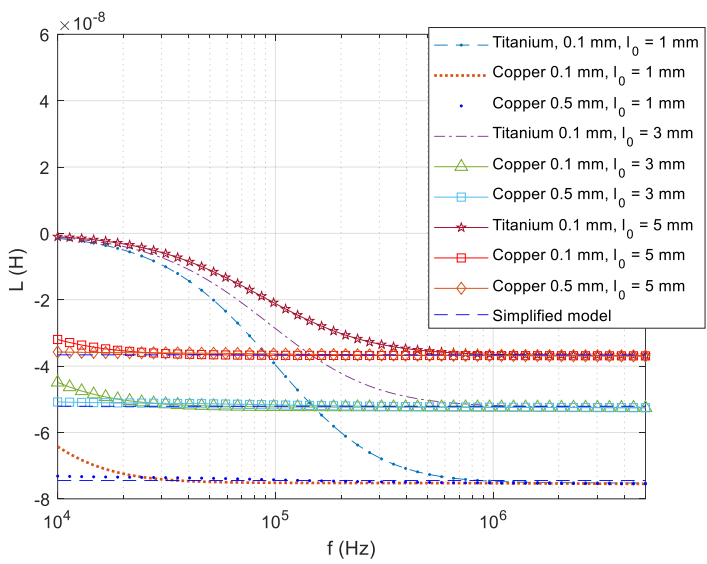

Fig. 3 Simulation of high-frequency inductance change for the eddy-current sensor (size of Table 1) above the Titanium (electrical conductivity of 2.4 $\mathrm{MS} / \mathrm{m}$ ) plate and copper plates with different thicknesses using both conventional formula in (1) and simplified analytical formula in (14)

To retrieve the lift-off information, it is necessary to analyse the coupling effect of different thicknesses. Titanium and copper are selected as the general limit of most non-magnetic samples. Fig. 3 shows the simulation of swept-frequency inductance change for the sensor above the Titanium (electrical conductivity of $2.4 \mathrm{MS} / \mathrm{m}$ ) plate and copper plates (electrical conductivity of $58.7 \mathrm{MS} / \mathrm{m}$ ) with different thicknesses. It can be found that under high working frequencies (over $1 \mathrm{MHz}$ ), the inductance computed from the conventional analytical model in (1) is insensitive to the thickness variations, and (converges at) matches well with the quiescent inductance from the simplified model in (14). Previously, due to the measurement uncertainty of the specimen (i.e. thickness effect), the Lissajous diagram [44] is proposed for the establishment of a lift-off curve under high frequencies using a calibration specimen of nominal thickness. Regarding the differing lift-off conditions, the proposed method is continuous in lift-off and thickness without calibration. The conventional "Lissajous" method employs a set of calibration specimens that span the thickness and lift-off range of interest to the application. The number of thicknesses and lift-off distances are chosen such that adequate accuracy is achieved using interpolation over a 2D calibration surface in R-L (resistance-inductance) space for the pair of impedance measurements. Hence, both methods deal with changing lift-off and thickness conditions for online application. However, for the potential high-temperature measurement (e.g. testing of hot strip steel mill [45]), the sensor dimensions (e.g. coil height, radius, spacing, etc.) will slightly vary with the temperature. Changing of sensor dimensions will result in different impedance responses and 2D R-L calibration diagrams. Thus, a changing step of each sensor dimensions (coil height, radius, spacing, etc.) can lead to a new trivial series of diagram mapping. The proposed method is analytically based, which can easily be adapted to sensors with different dimensions or varying dimensions (e.g. with different temperatures). Overall, the proposed embedded algorithms save time and effort, are inexpensive (as the calibration blocks are manufactured to the highest standard of quality), eliminate the 
calibration need (As potential human factors could highly affect the reliability of the NDT inspection in the calibration process). Examples of analytical based R-L space diagram for the inductive coil above the half-space conductor, tilted effect of sensors, and thin-skin regime of inductive coil above long surface crack model are reported in $[43,46,47]$. Moreover, with the analytical based algorithm, multiple properties (including thickness, magnetic permeability, electrical conductivity, and lift-off) could be simultaneously retrieved from multifrequency impedance [9, 26, 29] (e.g. the Newton-Raphson based inverse solver [29]). The capability of proposed method compared to [29] is only a single-frequency inductance is required for the lift-off retrieval. Besides, the lift-off range is much extended up to $12 \mathrm{~mm}$. However, the proposed method is limited to non-magnetic plates with an accurate measurement of electrical conductivity. The electrical conductivity of the test piece is derived from four-terminal measurement. With the simplified algorithm in (14), the lift-off distance is retrieved without the calibrated specimen and nominal thickness profile. Moreover, compared to the conventional formula in (1) (DoddDeeds model), the simplified (high-frequency regime) one is already after the integral. Therefore, the simplified algorithm can apply for the online lift-off retrieval as it only takes milliseconds (compared to the conventional formula in (1), which takes over 10 seconds due to the integration over the whole wavenumber - $\alpha$ domain).

\section{B. Measurement system and sensor setup}

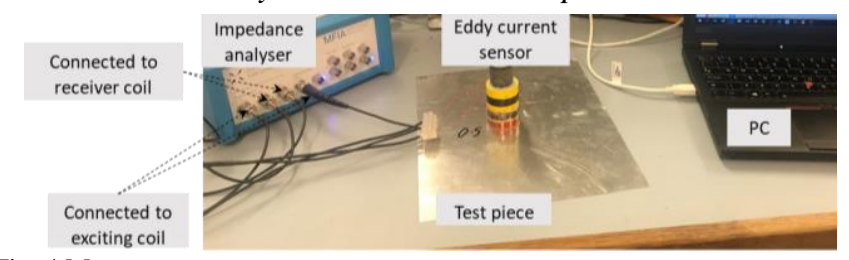

Fig. 4 Measurement system

For the experimental verification, the eddy current sensor is connected to the Zurich impedance analyser (Fig. 4). Considering the effect of non-ideal electromagnetic interferences, the measured data is refined by subtracting to the background/ambient signal (noise) when the sensor is in the free space (without the test piece). The refined measurement data, i.e. the inductance change due to the test piece can be used for further signal processing on the retrieval of sample parameters using analytical solvers in (14). The sensor consists of two co-axial coils, including the circular exciting coil and receiver coil. As listed in Table 1, the two coils have different radii but the same height, and are wound at the same lift-off plane. The coil is wound using the enamelled copper wire. To test the inductance under different lift-off distance, 12 spacers with a thickness of $1.0 \mathrm{~mm}$ are used between the sensor and test piece. Besides, the inductance is measured under multi frequencies to test the performance of the proposed method (for the prediction of both lift-off distance and thickness of the nonmagnetic conductive plate) under different working frequencies. The working frequency of the measurement ranges from 0.1 to $500 \mathrm{kHz}$. Since the measured inductance has been found sensitive to lift-off distance and almost nearly independent of the test piece, the higher-frequency inductance is used for the prediction of the lift-off distance. Moreover, as the inductance is less affected by the lift-off distance and more sensitive to the test piece (particular the thickness without concerning the eddy current skin effect), the lower-frequency inductance is applied for the further prediction of the thickness of non-magnetic conductive plates.

Since the thickness of the non-magnetic sample should be much smaller than the skin depth (can be calculated using equation 15), three non-magnetic thin plates - aluminium, copper, and brass with thicknesses of less than $1 \mathrm{~mm}$ have been used for the measurement. As listed in Table 2, the selected samples have a wide range of electrical conductivity, which aims to test the performance of the proposed prediction method for different properties of the (non-magnetic conductive) sample.

TABLE II

PROPERTIES OF NON-MAGNETIC SAMPLES

\begin{tabular}{cccc}
\hline \hline & Aluminium & Copper & Brass \\
\hline Electrical conductivity / $\mathrm{MSm}^{-1}$ & 36.9 & 58.7 & 15.9 \\
Thickness / mm & 0.5 & 0.8 & 0.4 \\
\hline \hline
\end{tabular}

\section{RESULTS AND DISCUSSIONS}

\section{A. Experimental result and analysis}

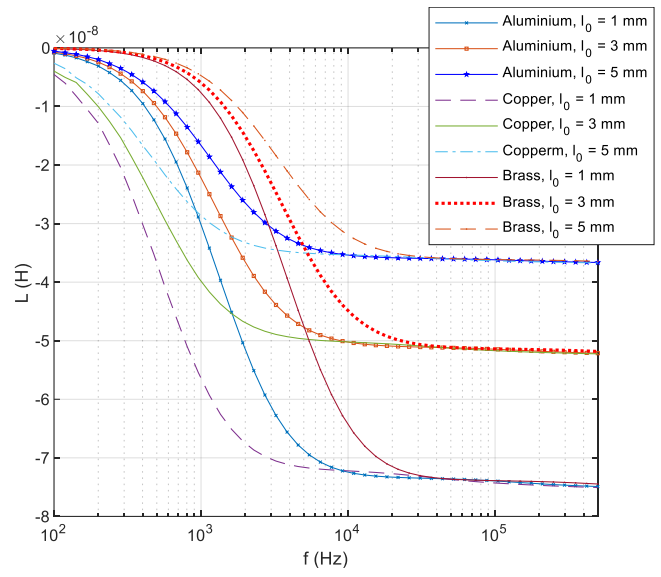

Fig. 5 Comparison of the measured inductance change (due to test piece) for different non-magnetic samples and lift-off distance (frequency ranges from 0.1 to $500 \mathrm{kHz}$ )

Fig. 5 shows the measured inductance change (due to test piece) of three samples under frequencies from 0.1 to $500 \mathrm{kHz}$ with different lift-off distances. The magnitude of the inductance curve increases rapidly and gradually nearly stable with the working frequencies owing to an enhanced coupled effect between the coils and test piece. It can be observed that for a specific lift-off distance, the inductance curve will shift laterally towards either left or right for different non-magnetic samples.

For a specific non-magnetic sample, the inductance curve is less affected by the lift-off distance between the eddy current sensor and test piece under lower working frequencies. Moreover, the inductance curve for a specific non-magnetic sample will significantly reduce with the lift-off (particularly under the high working frequency), which agrees with the liftoff decay factor $-\mathrm{e}^{-2 \alpha \mathrm{l}_{0}}$ in equation (1) (that is $\mathrm{e}^{-2 \alpha \mathrm{l}_{0}}$ approaches its maximum value -1 for a smaller lift-off value). Besides, it can be found that under the high working 


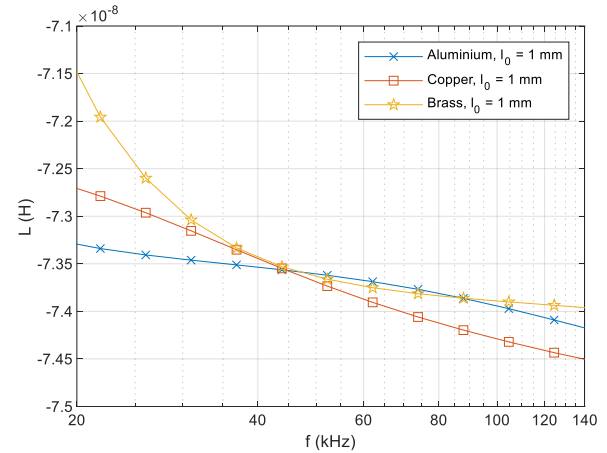

(a)

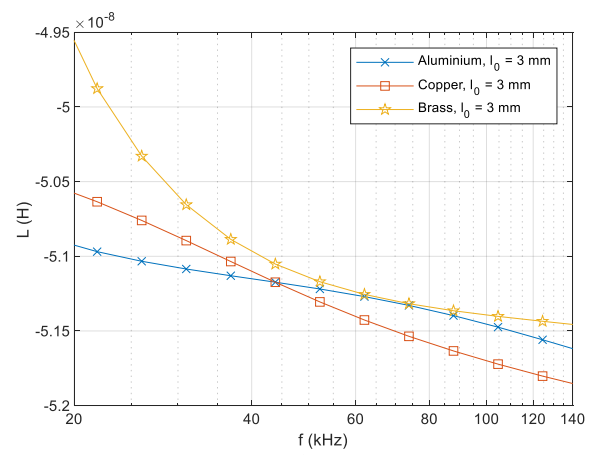

(b)

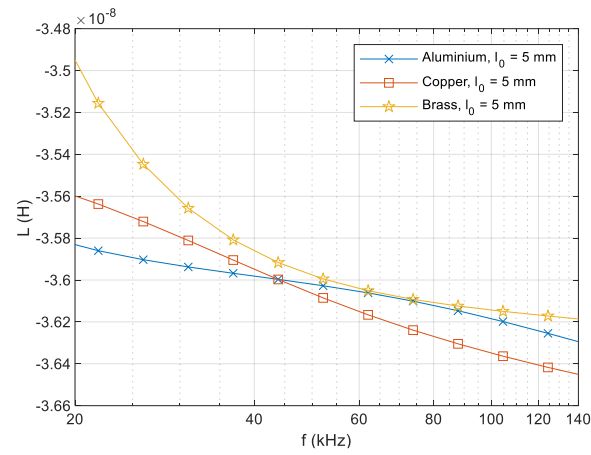

(c)

Fig. 6 Zooming-in comparison of the measured inductance change (due to test piece) for different non-magnetic samples (frequency ranges from 20 to $140 \mathrm{kHz}$ ) (a) lift-off distance of $1.0 \mathrm{~mm}$ (b) lift-off distance of $3.0 \mathrm{~mm}$ (c) lift-off distance of $5.0 \mathrm{~mm}$

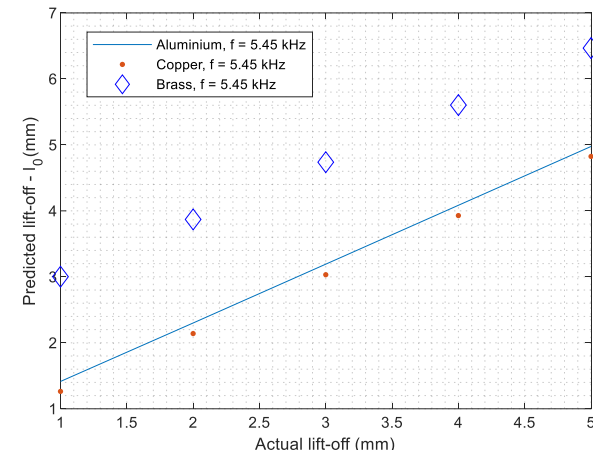

(a)

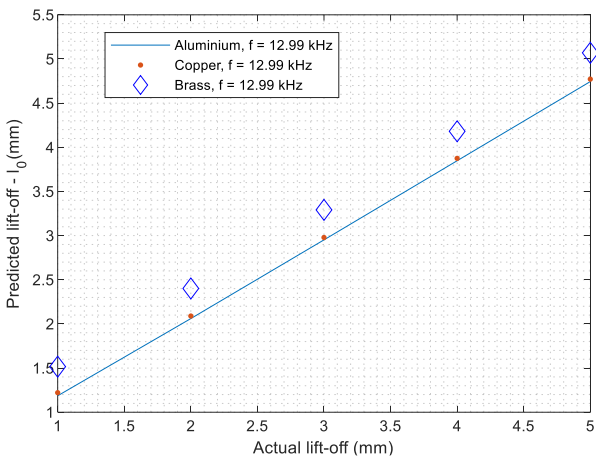

(b)

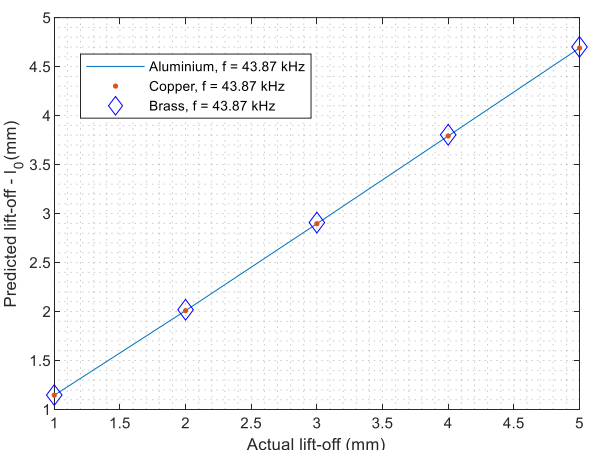

(c)

Fig. 7 Comparison of the predicted lift-off distance for different non-magnetic samples (lift-off ranges from 1.0 to $5.0 \mathrm{~mm}$ ) (a) under the working frequency of $5.45 \mathrm{kHz}$ (b) under the working frequency of $12.99 \mathrm{kHz}$ (c) under the working frequency of $43.87 \mathrm{kHz}$

frequencies, the inductance gradually becomes nearly independent of the test piece. The independence of the sample is due to the skin effect of the eddy current within the sample. The eddy current is constrained beneath the surface of the test piece under high working frequencies. Moreover, the highfrequency eddy current is almost delayed 180 degrees with respect to the induced voltage on the sensor. Therefore, the test piece can be treated as a pure inductor or inverter under high frequencies, which agrees with equation (12) $(\mathrm{T}=-1$ under high working frequencies).

Fig. 6 depicts the zooming-in frequency range for the inductance curves under a specific lift-off distance between the eddy current sensor and sample. It can be seen from Fig. 6 (a) that under the high working frequency and lift-off distance of $1.0 \mathrm{~mm}$, the difference between the inductance of three nonmagnetic samples gradually decreases and arrives at its lowest value (around $0.07 \%$ compared to the magnitude) around 43.87 $\mathrm{kHz}$. After $43.87 \mathrm{kHz}$, the difference of the inductance for three samples slightly increases and becomes a constant value with the working frequency. Similar trends can be observed for the lift-off distance of $3.0 \mathrm{~mm}$ in Fig. 6 (b) and $5.0 \mathrm{~mm}$ in Fig. 5 (c), where the different is around $0.28 \%$ and $0.25 \%$ respectively. Therefore, the optimal frequency for the prediction of the lift-off distance is around $43.87 \mathrm{kHz}$ for different non-magnetic samples.

\section{B. Prediction of lift-off distance}

As shown in Fig. 7, the lift-off distance has been predicted by finding the solution of the proposed non-linear equation in (14) under the working frequency of 5.45, 12.99, and 43.87 kHz. From (14), the lift-off distance can be estimated without the variables (parameters) of the test piece - electrical conductivity. It can be observed that for a small range of lift-off distance (from 1.0 to $5.0 \mathrm{~mm}$ ), the predicted lift-off distance is linear with but higher than the actual value under frequencies lower than $43.87 \mathrm{kHz}$. This is because the inductance curve is shown more sensitive to the test piece under lower frequencies (as depicted in Fig. 5). Besides, the proposed method in (14) is based on the high-frequency approximation $(\mathrm{T}=-1)$ where the inductance is nearly independent of the test piece. As the working frequency approaches $43.87 \mathrm{kHz}$, the prediction curve for three non-magnetic samples gradually overlaps (independent of test piece). For a small lift-off range up to 5.0 $\mathrm{mm}$, the maximum error of the predicted lift-off is around 0.3 $\mathrm{mm}$ (at $5.0 \mathrm{~mm}$ ) under $43.87 \mathrm{kHz}$.

Fig. 8 (a) illustrates the error of the lift-off prediction for a wide range up to $12.0 \mathrm{~mm}$ under the working frequency of $43.87 \mathrm{kHz}$. It can be found the error curves of the lift-off predicted from the inductance of three non-magnetic samples almost overlap on the whole lift-off range. Moreover, as the actual lift-off increases, the predicted lift-off begins from a slightly overestimated value and almost linearly varies to an underestimated value. The estimation error for a wide range of lift-off (up to $12.0 \mathrm{~mm}$ ) reaches its lowest value $-8.8 \mu \mathrm{m}$ at 2.0 $\mathrm{mm}$ and the highest value $-1.1 \mathrm{~mm}$ at $12.0 \mathrm{~mm}$ under $43.87 \mathrm{kHz}$.

It can be seen from Fig. 8 (b) that the maximum error of the restored lift-off becomes more sample-dependent but changes very slowly for frequencies higher than $43.87 \mathrm{kHz}$. Thus, any frequency higher than $43.87 \mathrm{kHz}$ is acceptable for lift-off retrieval. For thinner and less-conductive samples, the inductance curve shifts to higher frequencies [12]. Thus, the 


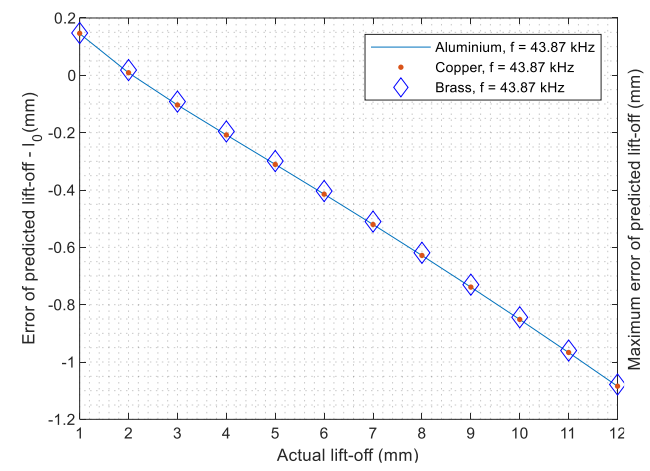

(a)

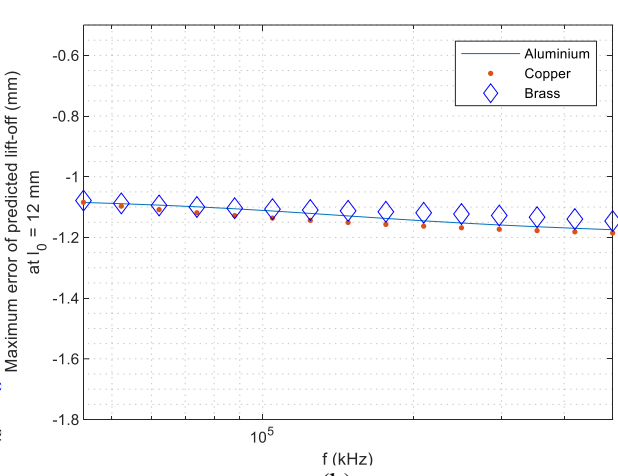

(b)

Fig. 8 (a) Comparison of the predicted lift-off distance for different non-magnetic samples (lift-off ranges from 1.0 to $12.0 \mathrm{~mm}$ ) under the working frequency of $43.87 \mathrm{kHz}$ (b) Maximum error of predicted lift-off at actual value of $\mathrm{l}_{0}=12 \mathrm{~mm}$ under different working frequencies

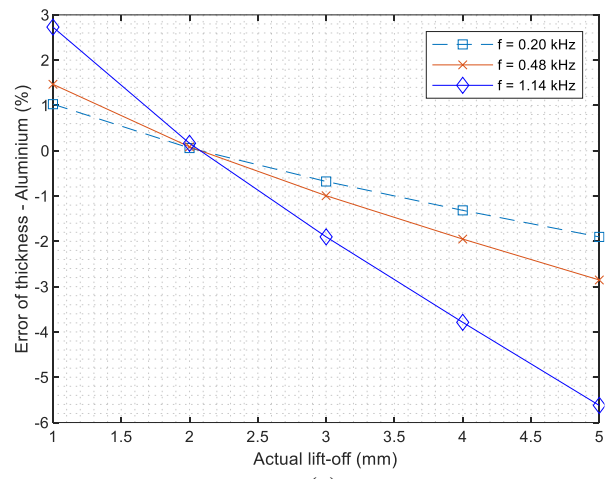

(a)

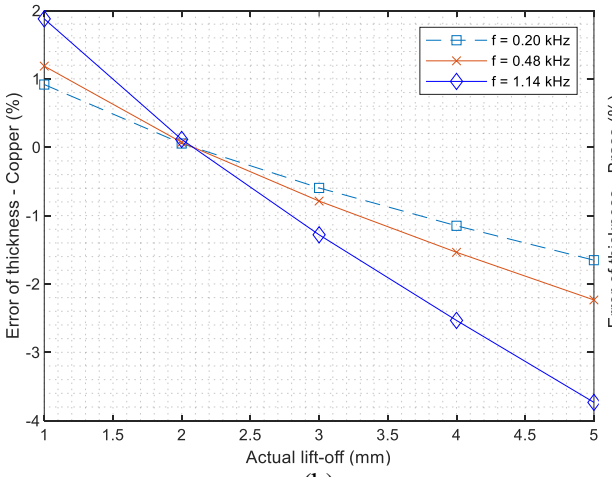

(b)

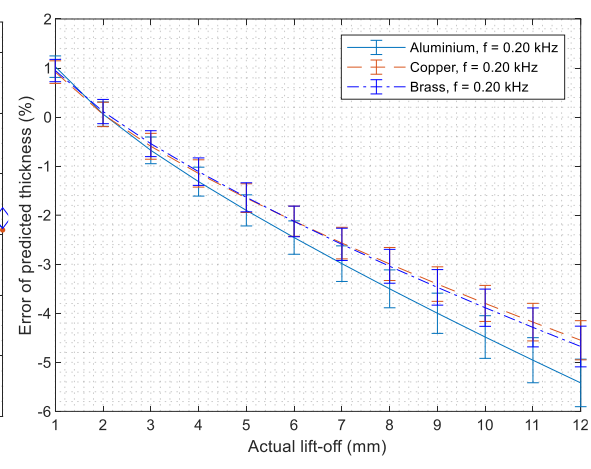

Fig. 10 Comparison for the error of the predicted thickness of different non-magnetic conductive plates (lift-off ranges from 1.0 to $12.0 \mathrm{~mm}$ ) under the working frequency of $0.20 \mathrm{kHz}$

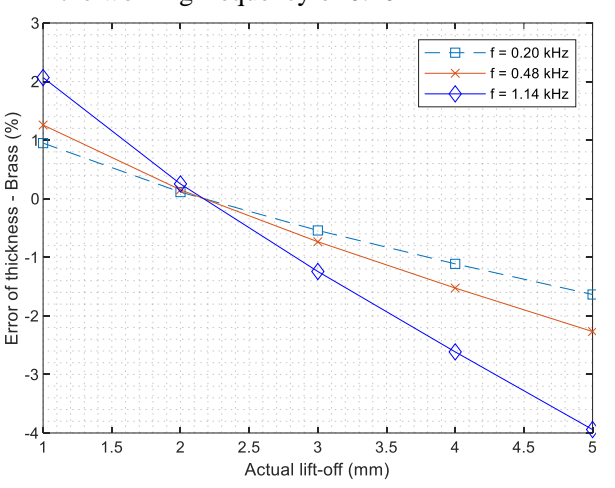

(c)

Fig. 9 Comparison for the error of the predicted thickness under different lower working frequencies. (lift-off ranges from 1.0 to $5.0 \mathrm{~mm}$ ) (a) Aluminium plate (b) Copper plate (c) Brass plate

minimum value of the optimal frequency range increases with reduced thickness (as can be observed from Fig. 3). Overall, for majorities of non-magnetic samples (with electrical conductivities range from 2.4 (Titanium) to $100 \mathrm{MS} / \mathrm{m}$ ), frequencies over $1 \mathrm{MHz}$ are within the optimal frequency range for the thickness of over $0.1 \mathrm{~mm}$.

\section{Prediction of sample thickness}

As shown in Fig. 9, the reconstructed lift-off can be used for the further prediction of the sample thickness. Parameters including the coil height, coil radii, number of turns (from the eddy current sensor in Table 1), electrical conductivity (from samples in Table 2), and reconstructed lift-off can be used for the initial reference value of the iterative method. The iterative loop can be terminated (under the condition/criterion) as the thickness change $\Delta \mathrm{c}$ in (17) becomes a stable residual.

Since the skin depth will decrease with the working frequency (according to equation 15) and may close to or even lower than the thickness of the test piece, the prediction of the sample thickness is operated under lower frequencies -0.20 , 0.48 , and $1.14 \mathrm{kHz}$. It can be seen from Fig. 8 that the prediction reaches its lowest error under the working frequency of 0.20 $\mathrm{kHz}$. This is because the inductance curve is more sensitive to the test piece and less affected by the lift-off distance under a lower working frequency (as can be seen from Fig. 5). Moreover, the skin depth for a lower working frequency is much larger than the thickness of the test piece, where the eddy current is more restrained by the bottom surface of the test piece. It can be seen that for a small lift-off range up to $5.0 \mathrm{~mm}$, the maximum error of the predicted sample thickness is around $2 \%$ (at $5 \mathrm{~mm}$ ) under $0.20 \mathrm{kHz}$.

Fig. 10 illustrates the error of the thickness prediction for a wide lift-off range up to $12.0 \mathrm{~mm}$ under the working frequency of $0.20 \mathrm{kHz}$. As the actual lift-off increases, the predicted thickness starts from an almost intersected and overestimated value, then becomes more sample-dependent and underestimated. Although the electrical conductivity is accurately measured (within $1 \%$ deviation of the actual value on the datasheet) through the four-terminal measurement method, error bars are included to present variations of the predicted thickness considering a maximum deviation of $10 \%$ of actual electrical conductivity (for three test pieces). As the error of the predicted lift-off reaches its lowest value at $2.0 \mathrm{~mm}$ (Fig. 8), the mean error of the predicted sample thickness achieved its lowest value $(0.11 \%)$ at the same actual lift-off 2.0 $\mathrm{mm}$. Besides, the maximum mean error of the predicted thickness is $5.42 \%$ at the lift-off distance of $12 \mathrm{~mm}$. Due to the different sensitivity of the inductance regarding the lift-off, the prediction error of the thickness differs from that of the lift-off distance. Moreover, the influence of conductivity variations on the predicted thickness is less than $1 \%$, which is due to the low sensitivity of inductance regarding the conductivity (More details on the multi-frequency sensitivities are reported in [26]). For the measurement uncertainty of electrical conductivity, impedance values under more different frequencies are required to simultaneously retrieve the thickness and electrical conductivity of test pieces $[9,26,29]$. 


\section{CONCLUSIONS}

This paper has introduced an algorithm for the measurement of the lift-off distance between the eddy current sensor and nonmagnetic conductive plate. According to the experimental result, the inductance for the sensor above the sample has been found sensitive to the lift-off and nearly independent of different non-magnetic samples (with an error of $0.28 \%$ ) under a single high frequency $43.87 \mathrm{kHz}$. Based on this effect (termed as the sample-independent insurance (SII) feature), the lift-off distance can be predicted with a maximum absolute error of 1.1 $\mathrm{mm}$ at the actual distance of $12.0 \mathrm{~mm}$. Moreover, it has been found that any frequency higher than $43.87 \mathrm{kHz}$ is acceptable for the lift-off retrieval. Considering the effect of measurement uncertainty (especially the thickness of the test piece), working frequencies over $1 \mathrm{MHz}$ apply to most non-ferrous metals with thicknesses of over $0.1 \mathrm{~mm}$.

Moreover, the predicted lift-off distance is used as the reference value for the prediction of the sample thickness. The prediction is operated under the iterative manner. Concerning the skin depth of the eddy current, a lower single frequency is used for the thickness prediction of the non-magnetic plates. From the measurement of different materials including aluminium, copper, and brass, the thickness can be accurately predicted with a maximum relative error of $5.42 \%$ at the liftoff distance of $12.0 \mathrm{~mm}$.

\section{REFERENCES}

[1]. K. J. Kirk, J. Elgoyhen, J. P. Hood, D. Hutson, R. S. Dwyer-Joyce, J. Zhang, and B.W. Drinkwater, "Ultrasonic condition monitoring using thin-film piezoelectric sensors," Insight-Non-Destructive Testing and Condition Monitoring, vol. 52, no. 4, pp. 184-191, 2010.

[2]. K. J. Kirk, A. McNab, A. Cochram, I. Hall, and G. Hayward, “ Ultrasonic arrays for monitoring cracks in an industrial plant at high temperatures," IEEE transactions on ultrasonics, ferroelectrics, and frequency control, vol. 46, no. 2, pp. 311-319, 1999.

[3]. J. Angelo, A. Bennecer, P. Picton, S. Kaczmarczyk, and A. Soares, "Eddy current analysis of shipped stainless steel heat exchanger bundle," Case Studies in Nondestructive Testing and Evaluation, vol. 6 (A), pp. 89-93, 2016.

[4]. A. Oyeniyan, A. Olamide, and A. Bennecer, "Fatigue Assessment of Welded Pipelines in Ultra-Deep Water," Abstract from COMSOL Conference, Lausanne, Switzerland, 2018.

[5]. I. N. Prassianakis, and N. I. Prassianakis, "Ultrasonic testing of nonmetallic materials: concrete and marble," Theoretical and Applied Fracture Mechanics, vol. 42, no. 2, pp. 191-198, 2004.

[6]. M. Kalms, O. Focke, and C. V. Kopylow, "Applications of laser ultrasound NDT methods on composite structures in aerospace industry," In Ninth International Symposium on Laser Metrology, vol. 7155, p. $71550 \mathrm{E}, 2008$.

[7]. T. Takagi, and K. Miya, "ECT round-robin test for steam generator tube," Journal of the Japan Society of Applied Electromagnetics, vol.8, pp. $121-8,2000$.

[8]. J. Moulder, C. E. Uzal, and J. H. Rose, "Thickness and conductivity of metallic layers from eddy current measurements," Review of scientific instruments, vol. 63, pp. 3455-3465, 1992.

[9]. Y. Nonaka, "A double coil method for simultaneously measuring the resistivity, permeability, and thickness of a moving metal sheet," IEEE Transactions on Instrumentation and Measurement, vol. 45, pp. 478-482, 1996.

[10]. D. Placko, H. Clergeot, and E. Santander, "Physical modelling of an eddy current sensor designed for real time distance and thickness measurement in galvanization industry," IEEE Transactions on Magnetics, vol. 25, pp. 2861-2863, 1989.
[11]. A. Sethuraman, and J. H. Rose, "Rapid inversion of eddy current data for conductivity and thickness of metal coatings," Journal of nondestructive evaluation, vol. 14, pp. 39-46, 1995.

[12]. W. Yin, and A. J. Peyton, "Thickness measurement of non-magnetic plates using multi-frequency eddy current sensors," NDT \& E International, vol. 40, no. 1, pp. 43-48, 2007.

[13]. S. Liu, Y. Sun, M. Gu, C. Liu, L. He, and Y. Kang, "Review and analysis of three representative electromagnetic NDT methods," Insight-NonDestructive Testing and Condition Monitoring, vol. 58, no. 4, pp. 17683, 2017.

[14]. Y. Li, G. Tian, and S. Ward, "Numerical simulation on magnetic flux leakage evaluation at high speed," NDT \& E International, vol. 39, no. 5, pp. 367-73, 2006.

[15]. D. Kim, L. Udpa, and S. Udpa, "Remote field eddy current testing for detection of stress corrosion cracks in gas transmission pipelines," Materials Letters, vol. 58, no. 15, pp. 2102-2104, 2004.

[16]. L. Udpa, S. Mandayam, S. S. Udpa, Y. S. Sun, and W. Lord, "Developments in gas pipeline inspection technology," Materials Evaluation, vol. 54, no. 4, pp. 467-472, 1996.

[17]. G. Katragadda, J. T. Si, W. Lord, Y. S. Sun, S. S. Udpa, and L. Udpa, "Alter-native magnetic flux leakage modalities for pipeline inspection," IEEE Transactions on Magnetics, vol. 32, no. 3, pp. 1581-1584, 1996.

[18]. W. Lord, S. Sun, S. S. Udpa, and S. Nath, "A finite element study of the remote field eddy current phenomenon," IEEE Transactions on Magnetics, vol. 24, no. 1, pp. 435-438, 1988.

[19]. M. Lu, L. Yin, A. J. Peyton, and W. Yin, "A novel compensation algorithm for thickness measurement immune to lift-off variations using eddy current method," IEEE Transactions on Instrumentation and Measurement, vol.65, no. 12, pp. 2773-9, 2016.

[20]. A. V. Egorov, V. V. Polyakov, D. S. Salita, E. A. Kolubaev, S. G. Psakhie, A. G. Chernyavskii, and I. V. Vorobei, "Inspection of aluminum alloys by a multi-frequency eddy current method," Defence Technology, vol. 11, no. 2, pp. 99-103, 2015.

[21]. T. Theodoulidis, and E. E. Kriezis, "Eddy current canonical problems (with applications to nondestructive evaluation)," Tech Science Press, no. 0-9717880-1-4, USA, 2006.

[22]. E. Pinotti, and E. Puppin, "Simple Lock-In Technique for Thickness Measurement of Metallic Plates," IEEE Transactions on Instrumentation and Measurement, vol. 63, no. 2, pp. 479-484, 2014.

[23]. M. Lu, X. Meng, W. Yin, Z. Qu, F. Wu, J. Tang, et al., "Thickness measurement of non-magnetic steel plates using a novel planar triple-coil sensor," NDT \& E International, vol. 107, 2019.

[24]. R. Huang, M. Lu, A. J. Peyton, and W. Yin, "Thickness measurement of metallic plates with finite planar dimension using eddy current method," IEEE Transactions on Instrumentation and Measurement, early access, 2020. Doi:10.1109/TIM.2020.2987413.

[25]. G. Tytko, and L. Dziczkowski, "E-Cored Coil With a Circular Air Gap Inside the Core Column Used in Eddy Current Testing," IEEE Transactions on Magnetics, vol. 51, no. 9, pp. 1-4, 2015.

[26]. W. Yin, A. J. Peyton, and S. J. Dickinson, "Simultaneous Measurement of Distance and Thickness of a Thin Metal Plate With an Electromagnetic Sensor Using a Simplified Model," IEEE Transactions on Instrumentation and Measurement, vol. 53, no. 4, pp. 1135-1138, 2004.

[27]. M. Lu, H. Xu, W. Zhu, L. Yin et al., "Conductivity Lift-off Invariance and measurement of permeability for ferrite metallic plates," NDT \& E International, vol. 95, pp. 36-44, 2018.

[28]. M. Lu, R. Huang, W. Yin, Q. Zhao, and A. J. Peyton, "Measurement of permeability for ferrous metallic plates using a novel lift-off compensation technique on phase signature," IEEE Sensors Journal, vol. 19, no. 17, pp. 7440-7446, 2019.

[29]. M. Lu, et al., "Determination of the magnetic permeability, electrical conductivity, and thickness of ferrite metallic plates using a multifrequency electromagnetic sensing system," IEEE Transactions on Industrial Informatics, vol. 15, pp. 4111-4119, 2019.

[30]. X. Chen, and Y. Lei, "Electrical conductivity measurement of ferromagnetic metallic materials using pulsed eddy current method," NDT \& E International, vol. 75, pp. 33-38, 2015. 
[31]. D. Vasic, V. Bilas, and D. Ambrus, "Pulsed eddy-current nondestructive testing of ferromagnetic tubes," IEEE Transactions on Instrumentation and Measurement, vol. 53, no. 4, pp. 1289-1294, 2004.

[32]. I. Z. Abidin, C. Mandache, G. Y. Tian, and M. Morozov, "Pulsed eddy current testing with variable duty cycle on rivet joints," NDT \& E International, vol. 42, no. 7, pp. 599-605, 2009.

[33]. Y. He, G. Tian, H. Zhang, M. Alamin, A. Simm, and P. Jackson, "Steel Corrosion Characterization Using Pulsed Eddy Current Systems," IEEE Sensors Journal, vol. 12, no. 6, pp. 2113-2120, 2012.

[34]. Y. Shin, D. Choi, Y. Kim, and S. Lee, "Signal characteristics of differential-pulsed eddy current sensors in the evaluation of plate thickness," NDT \& E International, vol. 42, no. 3, pp. 215-221, 2009.

[35]. D. Wen, M. Fan, B. Cao, B. Ye, and G. Tian, "Extraction of LOI Features from Spectral Pulsed Eddy Current Signals for Evaluation of Ferromagnetic Samples," IEEE Sensors Journal, vol. 19, no. 1, pp. 189195, 2019.

[36]. G. Y. Tian, and A. Sophian, "Reduction of lift-off effects for pulsed eddy current NDT," NDT \& E International, vol. 38, no. 4, pp. 319-324, 2005.

[37]. C. C. Tai, J. H. Rose, and J. C. Moulder, "Thickness and conductivity of metallic layers from pulsed eddy-current measurements," Review of Scientific Instruments, vol. 67, no. 11, 1996.

[38]. M. Lu, et al., "Acceleration of frequency sweeping in eddy-current computation,” IEEE Transactions on Magnetics, vol. 53, pp. 1-8, 2017.

[39]. W. Zhou, M. Lu et al., "Three-dimensional electromagnetic mixing models for dual-phase steel microstructures," Applied Sciences, vol. 8, p. 529, 2018.

[40]. G. Yang, G. Dib, L. Udpa, A. Tamburrino and S. S. Udpa, "Rotating Field EC-GMR Sensor for Crack Detection at Fastener Site in Layered Structures," IEEE Sensors Journal, vol. 15, no. 1, pp. 463-470, 2015.

[41]. W. Li, X. Yuan, G. Chen, J. Ge, X. Yin, and K. Li, "High sensitivity rotating alternating current field measurement for arbitrary-angle underwater cracks," NDT \& E International, vol. 79, pp. 123-131, 2016.

[42]. M. Lu et al., "Measurement of ferromagnetic slabs permeability based on a novel planar triple-coil sensor," IEEE Sensors Journal, vol. 20, no. 6, pp. 2904-2910, 2020.

[43]. C. V. Dodd, and W. E. Deeds, "Analytical solutions to eddy-current probe-coil problems," Journal of applied physics, vol. 39, no. 6, pp. 2829-2838, 1968.

[44]. V. S. Cecco, F. L. Sharp, and G. Van Drunen, "Eddy current testing; v 1, manual on Eddy current method," Chalk River Nuclear Laboratories, No. AECL-7523. CM-P00067661, 1981.

[45]. S. J. Dickinson, R. Binns, W. Yin, C. Davis, and A. J. Peyton, "The development of a multifrequency electromagnetic instrument for monitoring the phase transformation of hot strip steel," IEEE Transactions on Instrumentation and Measurement, vol. 56, no. 3, pp. 879-886.

[46]. T. Theodoulidis, "Analytical model for tilted coils in eddy-current nondestructive inspection," IEEE Transactions on Magnetics, vol. 41, pp. 2447-2454, 2005.

[47]. R. J. Ditchburn, S. K. Burke, and M. Posada, "Eddy-current nondestructive inspection with thin spiral coils: Long cracks in steel," Journal of Nondestructive Evaluation, vol. 22, no. 2, pp. 63-77, 2003.

[48]. M. Lu, L. Chen, X. Meng, R. Huang, A. Peyton and W. Yin, "Thickness measurement of metallic film based on a high-frequency feature of triplecoil electromagnetic eddy current sensor," IEEE Transactions on Instrumentation and Measurement, early access.

[49]. J. R. S. Avila, M. Lu, et al., "Accurate measurements of plate thickness with variable lift-off using a combined inductive and capacitive sensor," NDT \& E International, vol. 110, pp. 102202, 2020.

[50]. M. Lu, W. Zhu, L. Yin, A. J. Peyton, W. Yin and Z. Qu, "Reducing the lift-off effect on permeability measurement for magnetic plates from multifrequency induction data," IEEE Transactions on Instrumentation and Measurement, vol. 67, no. 1, pp. 167-174, Jan. 2018.

[51]. M. Lu, et al., "Measuring lift-off distance and electromagnetic property of metal using dual-frequency linearity feature," IEEE Transactions on Instrumentation and Measurement, early access, 2020. Doi: 10.1109/TIM.2020.3029348.

[52]. M. Lu, et al., "Lift-off tolerant pancake eddy-current sensor for the thickness and spacing measurement of nonmagnetic plates," IEEE
Transactions on Instrumentation and Measurement, early access, 2020. DOI: 10.1109/TIM.2020.3033377

[53]. W. Yin, J. Tang, M. Lu, et al., "An equivalent-effect phenomenon in eddy current non-destructive testing of thin structures," IEEE Access, vol. 7, pp. 70296-70307, 2019.

[54]. R. Huang et al., "Measuring Co-Axial Hole Size of Finite-Size Metallic Disk Based on a Dual-Constraint Integration Feature Using MultiFrequency Eddy Current Testing," IEEE Transactions on Instrumentation and Measurement, early access, 2020. DOI: 10.1109/TIM.2020.3026762

[55]. R. Huang et al., "Measurement of the radius of metallic plates based on a novel finite region eigenfunction expansion (FREE) method," IEEE Sensors Journal, early access, 2020. DOI: 10.1109/JSEN.2020.3009443

[56]. J. Tang et al., "A Novel Efficient FEM Thin Shell Model for BioImpedance Analysis," Biosensors, vol. 10, no. 6, pp. 69, 2020.

[57]. L. Chen, et al., "Textile Based Capacitive Sensor for Physical Rehabilitation via Surface Topological Modification," ACS Nano, vol. 14, no. 7, pp. 8191-8201, 2020. DOI: 10.1021/acsnano.0c01643

[58]. Z. Jin, et al., "Methods of Controlling Lift-off in Conductivity Invariance Phenomenon for Eddy Current Testing," IEEE ACCESS, vol. 8, pp. 2169-3536, 2020. DOI: 10.1109/ACCESS.2020.3007216.

[59]. J. Tang, et al., "Effect of frozen-thaw injury on cell membrane and bioimpedance," In 2020 IEEE International Instrumentation and Measurement Technology Conference (I2MTC), pp. 1-6. IEEE, 2020.

[60]. J. Tang, et al., "Bio-impedance spectroscopy for frozen-thaw of biosamples: Non-contact inductive measurement and finite element (FE) based cell modelling," Journal of Food Engineering, vol. 272, pp. 109784, 2020 .

[61]. H. Xu et al., "Imaging a weld cross-section using a novel frequency feature in multi-frequency eddy current testing," Insight-NonDestructive Testing and Condition Monitoring, vol. 61, no. 12, pp. 738 $743,2019$.

[62]. Y. Xie et al., "Novel Wearable Sensors for Biomechanical Movement Monitoring Based on Electromagnetic Sensing Techniques," IEEE Sensors Journal, vol. 20, no. 2, 2020. DOI: 10.1109/JSEN.2019.2943487

[63]. W. Yin et al., "Permeability invariance phenomenon and measurement of electrical conductivity for ferrite metallic plates," Insight-NonDestructive Testing and Condition Monitoring, vol. 61, no. 8, pp. 472 479, 2019.

[64]. M. Lu et al., "A model for the triboelectric nanogenerator with inductive load and its energy boost potential," Nano Energy, vol. 63, pp. 103883, 2019.

[65]. M. Lu et al., "Forward solver for deep earth exploration and induction logging using custom built Edge - Element FEM technique," Acta Geologica Sinica, vol. 93, pp. 302-304, 2019.

[66]. L. Chen et al., "Whole System Design of Wearable Magnetic Induction Sensor for Physical Rehabilitation," Advanced Intelligent Systems, vol. 1, no. 1, pp. 1900037, 2019.

[67]. Y. X et al., "A self-powered radio frequency (RF) transmission system based on the combination of triboelectric nanogenerator (TENG) and piezoelectric element for disaster rescue/relief," Nano Energy, vol. 54, pp. 331-340, 2018.

[68]. W. Yin et al., "Custom edge-element FEM solver and its application to eddy-current simulation of realistic $2 \mathrm{M}$-element human brain phantom," Bioelectromagnetics, vol. 39, no. 8, pp. 604-616, 2018.

[69]. L. Yin et al., "Detection of corrosion pits based on an analytically optimised eddy current sensor," Insight-Non-Destructive Testing and Condition Monitoring, vol. 60, no. 10, pp. 561-567, 2018.

[70]. W. Yin et al., "Acceleration of eddy current computation for scanning probes," Insight-Non-Destructive Testing and Condition Monitoring, vol. 60 , no. 10 , pp. $547-555,2018$.

[71]. W. Zhou et al., "Three-dimensional electromagnetic mixing models for dual-phase steel microstructures," Applied Sciences, vol. 8, no. 4, pp. 547-555, 2018

[72]. M. Lu, et al., "Determining the magnetic permeability of ferrite steel strip by a custom inversion method," In Proc. 12th ECNDT, pp. 1-8. 2018

[73]. J. Tang, et al., "Cellular structure analysis based on magnetic induction finite element method simulations and measurements," bioRxiv, pp. 275271, 2018. DOI: 10.1101/275271 
[74]. J.R.S. Avila, et al., "A novel dual modality sensor with sensitivities to permittivity, conductivity, and permeability," IEEE Sensors Journal, vol. 18, no. 1, pp. 356-362, 2017.

[75]. T. Yang, et al., "Level measurement for saline with a small surface area using high frequency electromagnetic sensing technique," Measurement, vol. 101, pp. 118-125, 2017.

[76]. M. Lu, et al., "Prediction of the asymptotical magnetic polarization tensors for cylindrical samples using the boundary element method," In 2015 IEEE Sensors Applications Symposium (SAS), pp. 1-4. IEEE, 2015.

[77]. W. Yin, et al., "Measurements of thickness for metallic plates with coaxial holes using a novel analytical method with the modified integration range," IEEE Transactions on Instrumentation and Measurement, 2015. DOI: 10.1109/ACCESS.2020.3035333

[78]. M. Lu, et al., "Inversion of distance and magnetic permeability based on material-independent and lift-off insensitive algorithms using eddy current sensor," IEEE Transactions on Instrumentation and Measurement, 2020. DOI: 10.1109/TIM.2020.3036099 